\title{
Deux espèces de DASYHELEA nouvelles pour le «Midi» méditerranéen : D. SENSUALIS Kieffer 1919 et D. LITHOtelmatiCA Strenzke 1951 (DIPTERA CERATOPOGONIDAE).
}

Nous rapportons à Dasyhelea sensualis Kieffer 1919 et Dasyhelea lithotelmatica Strenzke 1951, deux espèces de Ceratopogonidae provenant de prélèvements effectués dans divers rockpools et creux d'arbres (platanes et amandiers) du « Midi » méditerranéen.

Ces deux espèces appartiennent au groupe Dasyhelea versicolor Winnertz 1852, caractérisé par la présence de deux bandes mésonotales sombres parasaggitales, l'aspect punctiforme de la cellule radiale, l'effacement plus ou moins net de la bifurcation de $\mathbf{M}_{2}$, l'absence de fossette sensorielle sur le $3^{\circ}$ article des palpes, la pubescence des yeux, la structure binoduleuse des articles antennaires XI-XII-XIII des $\sigma^{*}$. Ce groupe comprend:
D. versicolor Winnertz 1852,
D. dufouri Laboulbène 1869 ,
D. sensualis Kieffer 1919,
D. bilineata Goetghebuer 1920,
D. geleiana Zilah 1932,
D. lithotelmatica Strenzke 1951.

D. versicolor est isolé des autres représentants du groupe par le rapport de longueur des articles 3 et 5 des palpes. Ce rapport est inférieur à 1 chez cette espèce, alors que chez toutes les autres il est supérieur à 1 .

En 1926, Edwards met en synonymie D. dufouri, D. bilineata et $D$. sensualis en se basant sur la similitude de couleur du mésonotum et la présence d'anneau sombre sur $\mathrm{T}_{1}$. Plus tard, Strenzke individualise à nouveau $D$. bilineata en insistant sur les différences d'alignement des papilles stigmatiques de la corne prothoracique de la nymphe. Au nom de l'écologie, il suit Edwards pour la synonymie de $D$. dufouri et $D$. sensualis.

Reconnaissons que les textes et figures des communications princeps sont pour la plupart trop imprécis, surtout en ce qui concerne les genitalia, pour séparer nettement ces espèces et éviter toute discussion taxonomique. Ainsi, Laboulbène figure les styles de $D$. dufouri à concavité externe, disposition qui n'a jamais été retrouvée. Au demeurant, si de légères différences de couleur ne justifient pas la création de nouvelles espèces, à l'inverse on ne peut accepter un regroupement sur de semblables critères. Tant que le type de $D$. dufouri ne sera pas redécrit, nous serons tenus de conserver $D$. sensualis.

En 1932, Zilah décrit une nouvelle espèce, D. geleiana, née de larves provenant de rockpools d'Allemagne. Dix-neuf ans plus tard, Strenzke décrit à son tour D. lithotelmatica 
à partir de larves issues, elles aussi, de rockpools d'eau douce. Il sépare son espèce de D. sensualis par la forme du style et les soies du scape et de D. geleiana par certains caractères nymphaux.

Nous rapportons à $D$. sensualis les adultes issus des gîtes de creux d'arbres et à $D$. lithotelmatica, ceux issus des gîtes de rochers. En effet, D. geleiana peut être facilement séparé de ces deux espèces par l'absence de grandes soies sensorielles sur les articles du flagellum $\uparrow$.
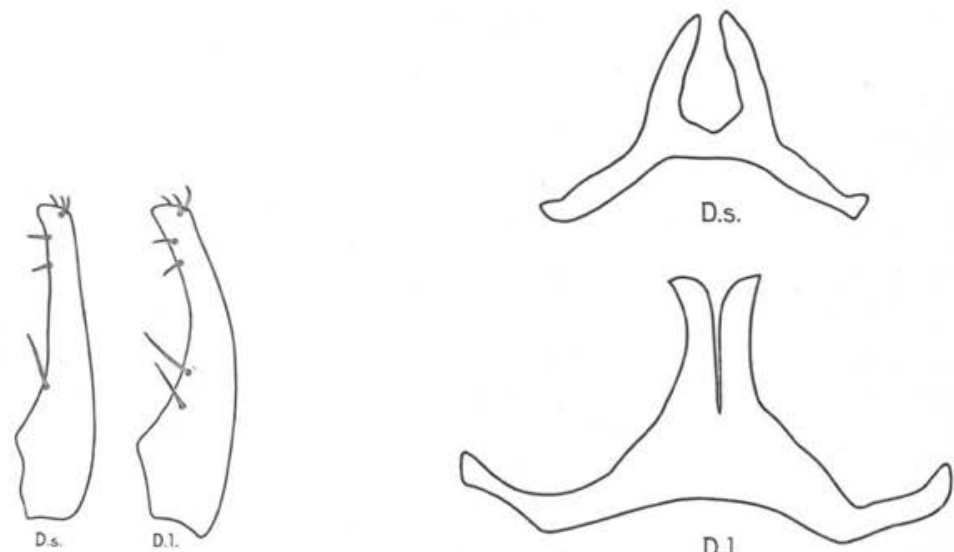

FIG. - Styles (à gauche) et aedagus (à droite) de Dasyhelea sensualis (D.s.) et Dasyhelea lithotelmatica (D.1.).

Quant à la distinction de $D$. lithotelmatica et $D$. sensualis, compte tenu des différences fondamentales de biotope larvaire, elle repose sur les deux caractères suivants :

$1^{\circ}$ le style des forcipules est courbe chez $D$. lithotelmatica et subrectiligne chez $D$. sensualis (fig. 1, A) ;

$2^{\circ}$ l'aedeagus présente une juxtaposition des processus paramédians chez $D$. lithotelmatica, ces processus restant nettement séparés dès leur base chez $D$. sensualis.

Localités de D. lithotelmatica Strenzke 1951:

La Fage (Hérault).

Saint-Gély-du-Fesc (Hérault).

La Baillaurie (P.-O.).

Localités de D. sensualis Kieffer 1919 :

Celleneuve (Hérault).

Maugio (Hérault).

Jean-A. Rioux et Suzanne Descous.

Laboratoire de Parasitologie, Faculté de Médecine, Montpellier. 


\section{Bibliographie}

Edwards (F. W.), 1926. - On the British biting Midges (Diptera, Ceratopogonidae). Trans. Entom. Soc., London, 74 (389-426).

Goetghebuer (M.), 1920. - Ceratopogoninae de Belgique. Mém. Mus. R. Hist. Nat. de Belgique, 8 (1-116).

- 1924. - Notes biologiques et morphologiques sur Dasyhelea bilineata Goetgh. Diptera, 1 , fasc. 3 (121-124).

- et Lenz (F.), 1934. - Heleidae (Ceratopogonidae) in Lindner's « Die Fliegen », Stuttgart, 133 p.

KiefFer (J. J.), 1919. - Chironomides d'Europe conservés au Musée National Hongrois de Budapest. Ann. Mus. Nat. Hung. Budapest, 17 (55-56).

- 1919. - Observations sur les Chironomides (Dipt.) décrits par J.-R. Malloch. Bull. Soc. Ent. France, 10 (191-194).

- 1925. - Faune de France. XI. Diptères (Nématocères piqueurs). Chironomidae Ceratopogonidae, Lechevalier, Paris, 138 p.

Laboulbène (A.), 1869. - Histoire des métamorphoses du Ceratopogon dufouri. Ann. Soc. Ent. Fr., (157-166).

Ohm (P.) et Remmert (H.), 1955. - Etude sur les rockpools des Pyrénées-Orientales. Vie et Milieu, 6 (204).

StrenzKe (K.), 1951. - Dasyhelea lithotelmatica n. sp. Archiv. für Hydrobiol., Sup. Band, 18 (178-187).

Zilah (von G.), 1932. - Anabiotische Dipteren. Arch. Hydrobiol., 23 (310-329). 